auch die Hill-Reaktion von Weizen-C mit $\left(\mathrm{Fe}(\mathrm{CN})_{6}\right)^{3 \ominus}$ als Elektronenakzeptor beträchtlich, um über $50 \%$, herabzudrücken. Damit ist er also kein echter Entkoppler der Phosphorylierungs-Reaktion (vgl. l. $\mathrm{c}^{13}$ ). Andererseits besitzen Vicia-C selbst die Fähigkeit zur Durchführung der H ill - Reaktion mit Reduktionsraten von $70-250 \mu$ Molen pro mg Chlorophyll in der Stde., ohne die zyklische PP in nennenswertem Umfange durchführen zu können. Wie die Verhältnisse hier im einzelnen liegen und welcher lipophile Stoff die Hemmwirkung der Vicia-C repräsentiert, bleibt nachzuprüfen. Ebenso ist die Frage ungelöst, welche Funktion der Hemmstoff in vivo hat. In diesem Zusammenhang sei darauf aufmerksam gemacht, daß die Höhe der PP von der Jahreszeit abhängig ist, ebenso wie die der Hill-Reaktion, deren diurnale Schwankungen bereits von HILL und SCARISBRICK ${ }^{14}$ aufgezeigt wurden:
Es gelang uns nicht, von November bis Februar gute PP-Raten bei Spinat und Winterweizen zu erhalten, während das gleiche Pflanzenmaterial vorher und nachher gute PP zeigte. Diese Befunde stehen in Übereinstimmung mit Beobachtungen von FücHTBAUER (private Mitteilung). Abb. 1 gibt einige repräsentative Daten für die PP über einen längeren Zeitraum wieder. Das Wiedereinsetzen der PP erfolgte mit dem Beginn des Wachstums bzw. dem Aufhören der Dormanzperiode. Auch die HillReaktion zeigte ähnliches Verhalten. Es erscheint möglich, daß der Hemmstoff physiologische Bedeutung für die Regulierung der an der PP beteiligten Elektronentransport-Prozesse hat.

Der Deutschen Forschungsgemeinschaft wird für die Unterstützung der Arbeiten gedankt. Fräulein M. Götre danke ich für ihre wertvolle Mitarbeit bei den Versuchen.

\title{
Zur unterschiedlichen Infektiosität von isolierter Ribonucleinsäure und intaktem Poliovirus in der Gewebekultur
}

\author{
Von Gebhard Koch \\ Aus dem Laboratorium der Stiftung zur Erforschung der spinalen Kinderlähmung \\ und der Multiplen Sklerose (Prof. Dr. H. PETTE), Hamburg-Eppendorf \\ (Z. Naturforschg. 15 b, 656-661 [1960] ; eingegangen am 14. Juli 1960)
}

\begin{abstract}
Tissue culture cells in monolayers do not exchange measurable amounts of cellulare ribonuclease with the surrounding medium under conditions used for assay of biological active ribonucleic acid. The low degree of infectivity of isolated ribonucleic acid from poliovirus is, therefore, not caused by degradation of the ribonucleic acid by cellulare ribonuclease.

Not all potentially infectious ribonucleic acid is taken up by the cells under standard assay conditions. Residual infectivity of ribonucleic acid in the supernatant of cell monolayers after incubation is considerably enhanced by a restoration of the hypertonicity of the solution.
\end{abstract}

McLaren, Holland und Syverton ${ }^{1}$ konnten den experimentellen Nachweis erbringen, daß auch die Infektion von Gewebekulturzellen durch Polioviren eingeleitet wird durch eine Adsorption der Viren an spezifische Rezeptoren der Wirtszellen, vergleichbar den ersten, bereits eingehend erforschten Stufen der Infektion von Bakterien durch Phagen ${ }^{2-4}$.

Bei der Darstellung infektiöser Ribonucleinsäure (RNS) aus intakten Polioviren ${ }^{5}$ verliert die RNS mit der Entfernung ihrer Proteinhülle auch ihr spezifisches Organ für die Adsorption an ihre Wirtszelle. Die nackte RNS ist zwar noch in der Lage,

1 L. C. Mclaren, J. J. Holland u. J. T. Syverton, J. exp. Medicine 109, 475 [1959]; J. J. Holland u. L. C. McLaren, J. exp. Medicine 109, 486 [1959].

2 A. D. Hershey, Advances in Virus Research 4, 25 [1957].

3 L. J. Tolmach, Advances in Virus Research 4, 63 [1957]. unter ganz bestimmten experimentellen Bedingungen eine Infektion der Wirtszellen zu induzieren, sie vermag aber diese nicht mehr mit dem Wirkungsgrad des kompletten Virusteilchens zu vollziehen. Es ist bisher noch nicht gelungen, in RNS-Präparaten mehr als $1 \%$ der Infektiosität des Virus-Ausgangsmaterials nachzuweisen. Wir hatten bereits in einer vorangegangenen Arbeit ${ }^{5}$ darauf hingewiesen, daß diese unterschiedliche Infektiosität von RNS und intaktem Virus wahrscheinlich durch eine niedere Empfänglichkeit der Wirtszellen für eine Infektion durch RNS und nicht durch eine Inaktivierung der RNS

4 A. Garen u. L. M. Kozloff, in: "The Viruses", Academic Press, New York, Vol. 2, 203 [1959].

5 G. Косн, S. Koenig u. H. E. Alexander, Virology 10, 329 [1960]. 
durch die zu ihrer Darstellung notwendigen Eingriffe hervorgerufen wird. Inzwischen konnten wir weiterhin zeigen, daß die RNS aus hochgereinigten Poliovirus-Suspensionen in guter Ausbeute isoliert werden kann (КосH, unveröffentlicht).

Es erscheint uns deshalb von Interesse, das Problem des unterschiedlichen Infektionsgrades von RNS und intaktem Virus durch weitere experimentelle Bearbeitung des Mechanismus der Infektion von Zellen durch RNS einer Lösung näher zu bringen. Wir haben vor kurzem über die Kinetik der Induktion von Plaques durch RNS in der Gewebekultur berichtet ${ }^{6}$. Es ergab sich dabei folgende Fragestellung:

1. Ist die Aufnahmefähigkeit der Gewebekulturzellen für RNS so gering, daß nur ein Bruchteil der potentiell infektiösen RNS nachweisbar wird?

2. Wird ein Teil der infektiösen RNS nach $\mathrm{Zu}$ gabe zu den Zellen durch noch vorhandene Ribonuclease inaktiviert?

3. Ist die aus Polioviren isolierte RNS heterogen in bezug auf ihr Reaktionsvermögen mit Zellen?

Zur Lösung dieser Problematik durchgeführte Experimente und deren Ergebnisse werden in dieser Arbeit beschrieben.

\section{Material und Methoden}

Wirtszellen: Als Wirtszellen für den PlaqueTest ${ }^{7}$ und für die Darstellung hochgereinigter und konzentrierter Viruspräparate verwendeten wir den permanenten Amnionzellstamm von Fernandes ${ }^{8}$ *. Die Zellen lassen sich gut in Suspension bei $37^{\circ} \mathrm{C}$ züchten. Wir verwenden dafür EAgLE's Medium ${ }^{9}$ mit einem $\mathrm{Zu}$ satz von $5-8 \%$ Kälberserum. Als Kulturgefäße benutzen wir runde $1 / 2-5-l$-Flaschen aus Jenaer Glas. Die Flaschen werden mit einem einfach oder doppelt durchbohrten und dann mit Watte wieder ausgefüllten Gummistopfen verschlossen. Das Ausmaß der Füllung der Flaschen ist in einem weiten Bereich $(1 / 10-9 / 10)$ ohne Einfluß auf die Zellvermehrung. Die Zellsuspension wird durch einen mit kontinuierlicher Drehzahlregelung versehenen Magnetrührer in ständiger Bewegung gehalten. Es empfiehlt sich, zwischen Magnetrührer und Kulturflasche eine ca. $0,5 \mathrm{~cm}$ starke Kork- oder Kunststoffplatte zu legen, um eine zu starke Erwärmung des

6 G. KocH, Experientia [Basel], im Druck.

7 R. Dulbecco u. M. Vogr, J. exp. Medicine 99, 167 [1954].

8 M. V. Fernandes, Texas Repts. Biol. and Med. 16, 48 [1958].

* Für die freundliche Uberlassung des Stammes möchten wir Herrn Dr. H. Moser, Biol. Laboratories, Long Island Biol. Ass., Cold Spring Harbor, L. I., N. Y., USA., herzlich danken.
Kulturgefäßes zu verhindern. Die Drehzahl des Rührstabes wird so eingestellt, $\mathrm{da} \beta$ die gesamte Suspension in ständiger Bewegung gehalten wird. Ein unerwünschtes breitflächiges Aufliegen des Rührstabes auf dem Glasboden läßt sich leicht vermeiden, wenn man den Rührstab in der Mitte etwa $1 \mathrm{~cm}$ breit mit einem PVCKunststoffschlauch überzieht. (Eine Härtung des Schlauches wird durch Aufbewahren des überzogenen Magnetstabes in 96-proz. Alkohol erreicht.) Für eine gleichbleibende Vermehrung der Zellen ist ein konstantes neutrales $p_{\mathrm{H}}$ unerläßlich. Wir vermeiden deshalb eine starke Verdünnung der Zellsuspension mit frischem Medium und züchten die Zellen bei einer Konzentration von $1-5 \cdot 10^{5}$ Zellen $/ \mathrm{ml}$.

Virus : Die Untersuchungen wurden mit der isolierten RNS von Poliovirus Typ I, Stamm Mahoney durchgeführt. Hochgereinigte Virussuspensionen wurden von uns im wesentlichen nach dem Verfahren von Levintow und Darnell ${ }^{10}$ dargestellt. Die Amnionzellen aus einer laufenden Suspensionskultur werden hierfür durch $10 \mathrm{~min}$ langes Zentrifugieren bei $700 \mathrm{U} / \mathrm{min}$ (Christ Universal III KS, großer Rotor) sedimentiert, einmal mit $\mathrm{E}$ agle's Medium gewaschen und danach 20-40-fach konzentriert in E a g le's Medium ohne Serum aufgenommen. Die Zellen werden mit 5 Plaque bildenden Einheiten/Zelle infiziert und $20 \mathrm{Stdn}$. bei $36{ }^{\circ} \mathrm{C}$ inkubiert und während dieser Zeit durch einen Magnetrührer in ständiger Suspension gehalten. Gröbere Zelltrümmer werden danach durch 30 min langes Zentrifugieren bei $3000 \mathrm{U} / \mathrm{min}$ abzentrifugiert und die Virusteilchen aus diesem Uberstand durch 2 Stdn. langes Zentrifugieren bei $40000 \mathrm{U} / \mathrm{min}$ (Spinco) sedimentiert. Das Sediment resuspendieren wir in 0,02-m. PhosphatPuffer $p_{\mathrm{H}} 7,1$, nicht in Lösung gehende Anteile werden durch niedertouriges Zentrifugieren entfernt und verworfen, die Virusteilchen erneut bei $40000 \mathrm{U} / \mathrm{min}$ sedimentiert, in Phosphatpuffer resuspendiert, von unlöslichen Anteilen befreit und abschließend durch Chromatographie über eine Säule aus Ecteola * gereinigt.

Darstellung der RNS: Wie bereits beschrieben ${ }^{5}$.

Lösungen : Als Standard-Lösung verwenden wir eine mit 0,01-m. Tris gepufferte Salzlösung, die alle Salze aus E a g l e's Medium (ausgenommen $\mathrm{NaHCO}_{3}$ ) in den entsprechenden Konzentrationen enthält (TBS). Zum Waschen der Zellkulturen in Petrischalen wird 0,7-m. $\mathrm{NaCl}$ enthaltende TBS, $p_{\mathrm{H}} 7,6$, zum Beimpfen der Kulturen mit RNS eine 1,1-m. $\mathrm{NaCl}$ enthaltende TBS $\left(p_{\mathrm{H}} 8,0\right)$ benutzt.

Biologischer Test: Die Bestimmung der Infektiosität von RNS und Viruspräparaten erfolgte nach dem Verfahren von Dulbecco und Vogt ${ }^{7}$. Für die Herstellung von Einschichten-Zellkulturen für den Pla-

9 H. EAGLE, Science [Washington] 130, 432 [1959].

10 L. Levintow u. J. E. Darnele, jr., J. biol. Chemistry 235, 70 [1960].

Herrn Dr. Levintow und Herrn Dr. Darnell danken wir für wertvolle Hinweise und für Mitteilung unveröffentlichter Befunde.

* Calif. Corporation for Biochem. Research. 
que-Test werden Zellen aus einer Suspensionskultur benutzt. Die Zellen werden abzentrifugiert, in $\mathrm{E}$ a g l e's Medium mit Calcium und einem Zusatz von $10-12 \%$ Kälberserum aufgenommen und mit einer automatischen Abfüllspritze auf 55-mm-Petrischalen verteilt. Jede Petrischale (Platte) erhält etwa $1,5 \cdot 10^{6}$ Zellen in $5 \mathrm{ml}$ Medium. Die Petrischalen werden in geschlossenen Plastikkästen $2-3$ Tage bei $37^{\circ} \mathrm{C}$ inkubiert. Ein konstantes $p_{\mathrm{H}}$ von $7,2-7,4$ wird erreicht durch Zugabe einer offenen Schale mit gesättigter $\mathrm{NaHCO}_{3}$-Lösung, die unmittelbar vor dem Verschließen des Plastikkastens mit einer kleinen Probe $\mathrm{CO}_{2}$-Trockeneis beschickt wird.

B e impfung der Gewebekultur-Plat ten (Standard-Testverfahren) : Bevor je 3 oder 4 Einschichten-Zellkulturen mit je 0,1-ml- Proben einer gegebenen RNS- oder Viruslösung beimpft werden, wird das Nährmedium abgesaugt und die Zellen werden mit einer gepufferten 0,7-m. Kochsalzlösung gewaschen $\left(p_{\mathrm{H}} 7,6\right)$. Die Zellkulturen werden nach dem Beimpfen $20 \mathrm{~min}$ bei $37{ }^{\circ} \mathrm{C}$ inkubiert und dann überschichtet mit $4 \mathrm{ml}$ Agarmedium folgender Zusammensetzung: 2 Tle. 4-proz. Difco-Agar in physiologischer Kochsalzlösung und 5 Tle. E a g l e's Medium mit 5\% Serum werden gemischt und Neutralrot bis zur Konzentration von 1 : 40000 zugegeben. Durch Zugabe von Neutralrot zum ersten Agar"overlay" wird gegenüber unserem früheren Verfahren ${ }^{5}$ ein Arbeitsgang eingespart, da die nachträgliche Zugabe von Neutralrot mit einer zweiten Agarlösung entfällt. Bei unserem jetzigen Verfahren muß die Zugabe der ersten Agarlösung jedoch bei gedämpftem Licht erfolgen, da Neutralrot unmittelbar nach Zugabe in Gegenwart von Licht toxisch für die Zellen ist ${ }^{11}$. Die beimpften Kulturen werden unter den gleichen Bedingungen wir vor der Beimpfung bei $37^{\circ} \mathrm{C}$ inkubiert. Die Plaques werden nach 2 und nach 3 Tagen gezählt.

Successive Beimpfung von mehreren Gewebekulturplatten mit einer RNS. Lös u n g : Mit einem bestimmten Volumen $(0,3$ bis $0,8 \mathrm{ml}$ ) einer RNS-Lösung wird innerhalb von $10 \mathrm{~min}$ eine Serie von 5 Platten successive beimpft, indem der RNS-Überstand einer Platte alle 2 min auf eine weitere Platte überführt wird. In einem Experiment werden jeweils 3 oder 4 Serien von je 5 Platten nebeneinander beimpft. Der nach $10 \mathrm{~min}$ von der 5. Platte entnommene Überstand wird auf die übliche Weise und nach Zugabe von $1 / 10$ Volumen $5-m$. $\mathrm{NaCl}$ auf verbleibende Infektiosität getestet.

\section{Ergebnisse}

In dem von uns beschriebenen Testsystem beträgt die Infektion der RNS $0,1-1 \%$ der des Virusausgangsmaterials ${ }^{5,12}$. Das Verhältnis von infektiösen zu physikalischen Teilchen liegt bei unseren Poliovirus-Suspensionen zwischen 1:100 und

11 S. H. Goodgal, persönliche Mitteilung.

12 A. Boeye, Virology 9, 691 [1959].

13 A. W. Rawin, Ann. Rev. Microbiol. 12, 309 [1958].
$1: 1000$. Da die RNS von Polioviren in nahezu $100 \%$ Ausbeute gewonnen werden kann, findet sich in unseren RNS-Präparaten nur eine infektiöse Einheit unter $10^{5}-10^{6}$ Polio-RNS-Äquivalenten. Frühere Befunde lassen vermuten, daß ein Plaque unter unseren Testbedingungen durch ein RNS-Molekül induziert werden kann ${ }^{5}$.

Die Zellschicht in den von uns für den PlaqueTest benutzten $55-\mathrm{mm}$-Petrischalen wird durch etwa $3-4 \cdot 10^{6}$ Zellen gebildet. Auf einer solchen Gewebekulturplatte können $700-800$ Plaques 18 bis 24 Stdn. nach der Beimpfung gerade noch erkannt werden. Nach Beimpfen mit stärker konzentrierten RNS-Lösungen beobachtet man zur gleichen Zeit eine konfluierende Zerstörung der Zellschicht. Also können weit mehr als 800 Plaques auf einer Platte durch RNS induziert werden. Es bleibt noch zu klären, ob alle Zellen einer Einschichtenkultur zu einer gegebenen Zeit durch RNS infiziert werden können oder ob - analog zu der DNS-Transformation von Bakterien in nicht synchronisierten Kulturen ${ }^{13}$ immer nur ein Teil der Zellen zur Aufnahme von RNS induziert werden kann.

Die Kinetik der Induktion von Plaques durch RNS in der Gewebekultur läßt sich nicht durch eine Empfänglichkeit nur weniger Zellen für eine Infektion durch RNS erklären. Diese Ansicht wird auch unterstüzt durch den Befund, daß die Anzahl der Plaques proportional zur zugegebenen RNS-Menge ist. Der Verlauf der Kinetik der RNS-Infektion kann dennoch durch die Zellen bestimmt werden. Es ist denkbar, daß die durch hypertone Salzlösung zur Aufnahme hochmolekularer Substanzen (Pinocytose $)^{5}$ angeregten Zellen nur für eine gewisse Zeit zur Aufnahme befähigt sind und daß ihre Reaktionsbereitschaft gegenüber RNS nach und nach abnimmt. Entsprechende Befunde wurden bei der Aufnahme von Proteinen durch Amöben mittels Pinocytose erhoben ${ }^{14,15}$. Es ist weiterhin denkbar, daß diese abnehmende Reaktionsbereitschaft durch Aufnahme inaktiver RNS bedingt wird, wenn nämlich die Aufnahme von RNS allgemein die Bereitschaft der Zellen für eine weitere Aufnahme von RNS herabsetzt.

Ist nun die geringe Infektiosität der RNS weitgehend durch die Zellen bestimmt, so ist zu erwarten, daß eine Vermehrung der Zellzahl/gegebene RNS-Menge zu einer Erhöhung der Plaque-Zahl

14 P. W. Brandt, Exp. Cell Res. 15, 300 [1958].

15 V. N. Schumaker, Exp. Cell Res. 15, 314 [1958]. 
führt. Vorausgesetzt, daß ein großer Teil der RNSMoleküle potentiell infektiös ist und die RNS in Lösung nicht durch Zellbestandteile inaktiviert wird, so ist bei einer successiven Beimpfung von Gewebekulturen zu erwarten, daß die gleiche Anzahl von Plaques/Platte gefunden wird, weitgehend unabhängig davon, wieviel Platten bereits mit der gegebenen RNS-Lösung beschickt worden sind.

$$
\begin{gathered}
\text { Successive Beimpfung } \\
\text { von Petrischalenkulturen } \\
\text { mit RNS-Lösungen }
\end{gathered}
$$

Um einer Lösung der oben erwähnten Problematik näher zu kommen, haben wir zuerst folgende Versuchsanordnung getroffen: Eine Reihe von 5 Gewebekulturplatten wurde in Abständen von 2 min nacheinander mit derselben RNS-Lösung beschickt. $0,1 \mathrm{ml}$ des Überstandes der 5. Platte wurde auf einer neuen Platte durch weitere 15 min lange Inkubation auf verbleibende Infektiosität getestet.

\begin{tabular}{|c|rr|rr|rr|rr|r|r|}
\hline & \multicolumn{8}{|c|}{ Volumen der RNS-Lösung } \\
Plat- & \multicolumn{2}{|c|}{$0,3 \mathrm{ml}$} & \multicolumn{2}{|c|}{$0,5 \mathrm{ml}$} & \multicolumn{2}{|c|}{$0,5 \mathrm{ml}$} & \multicolumn{2}{|c|}{$0,8 \mathrm{ml}$} \\
ten & $\mathrm{a}$ & $\mathrm{b}$ & $\mathrm{a}$ & $\mathrm{b}$ & $\mathrm{a}$ & $\mathrm{b}$ & $\mathrm{a}$ & $\mathrm{b}$ \\
\hline 1 & 23 & 59 & 106 & 47 & 262 & 49 & 40 & 29 \\
2 & 6 & 15 & 91 & 40 & 188 & 35 & 48 & 34 \\
3 & 9 & 23 & 22 & 10 & 76 & 14 & 35 & 25 \\
4 & 0 & 0 & 6 & 3 & 8 & 1,5 & 9 & 6 \\
5 & 1 & 3 & 1 & 0,5 & 0,7 & 0,1 & 9 & 6 \\
6 & 0 & & 0 & & 0 & & 2 \\
\hline
\end{tabular}

Tab. 1. Abnahme der Plaque-Zahl bei successiver Beimpfung von Gewebekulturen. a) Durchschnittliche Anzahl Plaques/Platte. b) \% Plaques vom Total der Platten $1-5$.

Die Ergebnisse dieser Untersuchung sind in Tab. 1 zusammengefaßt. Die Anzahl der durch RNS induzierten Plaques nimmt mit Übertragung von Platte zu Platte ab, bei Benutzung kleinerer Volumina scheinbar schneller als bei Beimpfung mit größeren Ausgangsvolumina. Die Ergebnisse stehen in scheinbarem Widerspruch zu unserer schon früher mitgeteilten Hypothese, daß die niedere Infektiosität der RNS im Vergleich zu intaktem Virus durch eine geringe Empfänglichkeit der Zellen bedingt ist. Die Möglichkeit, daß die Zellen die infektiöse RNS bereits nach kurzem Kontakt auf 3 Platten erschöpfend aufgenommen haben, kann ausgeschlossen werden, da bei der oben genannten Versuchsanordnung einer successiven Beimpfung von Kulturen mit 0,5 oder $0,8 \mathrm{ml}$ einer RNS-Lösung nur etwa $20-60 \%$ der im gewöhnlichen Testverfahren bestimmten Plaquezahl wiedergefunden wurde (Tab. 2).

\begin{tabular}{|c|c|c|c|}
\hline & \multicolumn{3}{|c|}{ Plaque bildende Einheiten } \\
Volumen der & im Standard- & $\begin{array}{c}\text { bei succes- } \\
\text { siver } \\
\text { RNS-Lösung }\end{array}$ & $\begin{array}{c}\text { Differenz } \\
\text { in \% }\end{array}$ \\
\hline 0,5 & 640 & 226 & 64 \\
0,5 & 940 & 535 & 43 \\
0,8 & 656 & 141 & 79 \\
\hline
\end{tabular}

Tab. 2. Vergleich der Ausbeute an Plaque bildenden Einheiten im Standard-Test-Verfahren und bei successiver $\mathrm{Be}$ impfung von 5 Gewebekulturen. Die letzte Spalte zeigt den Anteil von Plaque bildenden Einheiten, der bei successiver Beimpfung von 5 Platten nicht erfaßt wird, gemessen an der in Spalte 1 aufgeführten Anzahl von Plaque bildenden Einheiten, die im Standard-Test-Verfahren ermittelt wurden.

Da die Infektiosität der RNS-Lösung mit zunehmender Zellzahl nicht - wie erwartet - zunimmt, sondern im Gegenteil abnimmt, ist anzunehmen, daß zelluläre Bestandteile die Infektiosität der RNS beeinträchtigen. In weiteren Untersuchungen war zu prüfen, ob die Inaktivierung der RNS durch zelluläre Ribonuclease bedingt ist.

\section{Test auf zelluläre Ribonuclease}

Die bei der zuvor beschriebenen Versuchsanordnung der successiven Beimpfung einer Serie von Zellkulturen von der 5. Platte entnommene Lösung sollte, wenn sie Ribonuclease enthält, auch in der Lage sein, weitere aktive RNS zu inaktivieren. Wir haben deshalb den inaktiven Überstand der 5. Platte im Verhältnis 1:1 mit einer aktiven RNS-Lösung gemischt und die Stabilität der Infektiosität dieser Lösung geprüft. Bei 2-stdg. Inkubation bei $20^{\circ} \mathrm{C}$ zeigt sich keine Abnahme der Infektiosität, ebenso bei 2-wöchiger Inkubation bei $+4{ }^{\circ} \mathrm{C}$. Das Vorhandensein von Ribonuclease im Testsystem kann somit mit großer Wahrscheinlichkeit ausgeschlossen werden. Diese Auffassung wird durch weitere Ergebnisse unterstützt, auf die im folgenden eingegangen werden soll.

Ursache des Infektiositätsverlustes von RNS-Lösungen nach Kontakt mit mehreren Zellkulturen

Die biologische Aktivität isolierter Virusnucleinsäure in Gewebekulturplatten ist stark abhängig vom $p_{\mathrm{H}}$ der Lösung und deren Salzkonzentration. Die Infektiosität der RNS steigt mit der Erhöhung der Salzkonzentration und ist optimal an der Toxizitätsgrenze der Zellen für hypertone Lösungen. Bei einigen permanenten Zellstämmen wird durch hypertone Lösung eine Zellschrumpfung ausgelöst ${ }^{5}$, die so 
stark ist, daß sie mikroskopisch verfolgt werden kann. Scheinbar setzen die Zellen sich gegen die Hypertonie des umgebenden Mediums zur Wehr, in dem sie intrazelluläre Gewebeflüssigkeit nach außen abgeben.

Bei der successiven Beimpfung von Gewebekulturen beobachteten wir eine laufende Volumenzunahme der Lösung, die bis zu $60 \%$ der zur ersten Platte zugegebenen Lösungsmenge ausmachte. Diese Volumenzunahme kann nicht durch zurückgebliebene Waschflüssigkeit verursacht worden sein. Wir bemühen uns, überstehende Flüssigkeit stets so vollständig wie möglich zu entfernen. Eine Verdünnung der hypertonen Lösung verursacht jedoch eine bedeutende Abnahme der Infektiosität der RNS ${ }^{5}$.

Die durch Abgabe von intrazellulärer Gewebeflüssigkeit bewirkte Verdünnung der Salzkonzentration könnte demnach die Ursache dafür sein, daß sich in der RNS-Lösung nach mehrfachem Kontakt mit Zellkulturen keine Infektiosität mehr nachweisen läßt. Wie unsere in Tab. 3 zusammengefaßten Be-

\begin{tabular}{|c|c|c|}
\hline \multirow{2}{*}{$\begin{array}{l}\text { Expe- } \\
\text { riment }\end{array}$} & \multicolumn{2}{|c|}{$\begin{array}{l}\text { Durchschnittliche Anzahl } \\
\text { Plaques/Platte }\end{array}$} \\
\hline & $\begin{array}{l}\text { vor Zugabe } \\
\text { von } \mathrm{NaCl}\end{array}$ & $\begin{array}{l}\text { nach Zugabe } \\
\text { von } \mathrm{NaCl}\end{array}$ \\
\hline 1 & 1,3 & 63 \\
\hline 2 & 0 & 69 \\
\hline 3 & 2 & 30 \\
\hline 4 & 1,5 & 72 \\
\hline
\end{tabular}

Tab. 3. Vergleich der Infektiosität im Uberstand nach successiver Beimpfung von 5 Zellkulturen mit $0,8 \mathrm{ml}$ RNS-

Lösung vor und nach Zugabe von $1 / 10$ Volumen 5-m. NaCl.

funde zeigen, tritt die Infektiosität sofort wieder in Erscheinung, wenn die von den Zellkulturplatten entnommene Lösung wieder hyperton gemacht wird. Nach erneuter Zugabe von $\mathrm{NaCl}$ wird im Überstand der 5. Platte unter Einrechnung der Verdünnung nahezu die gleiche Infektiosität gefunden wie in der Ausgangslösung vor der successiven Beimpfung. Es war deshalb zu prüfen, ob auch nach Ablauf der Inkubation im Standard-Testverfahren verbleibende infektiöse RNS in der Beimpfungsflüssigkeit nachgewiesen werden kann.

Nachweis von verbleibender

RNS-Infektiosität nach Inkubation i m Standard-Testverfahren

Eine Verdünnung der hypertonen RNS-Lösung durch die Gewebekultur läßt sich auch im StandardTestverfahren nachweisen. Nach Beimpfen der Plat- ten mit $0,1 \mathrm{ml}$ einer 1,1-m. RNS-Lösung können nach 20 min Inkubation bei $37^{\circ} \mathrm{C}$ ca. $0,16-0,17 \mathrm{ml}$ Lösung entnommen werden. Wird diese Lösung unmittelbar nach Entnahme auf eine weitere Platte übertragen, so zeigt sie nur eine geringe oder keine Infektiosität. Nach erneuter Zugabe von $\mathrm{NaCl}$ läßt sich wieder eine beträchtliche Infektiosität nachweisen (Tab. 4).

\begin{tabular}{|c|c|c|c|}
\hline \multirow[b]{2}{*}{ Experiment } & \multicolumn{3}{|c|}{ Durchschnittliche Anzahl Plaques/Platte } \\
\hline & 1. Test & $\begin{array}{c}\text { vor Zugabe } \\
\text { von } \mathrm{NaCl}\end{array}$ & $\begin{array}{c}\text { nach Zugabe } \\
\text { von } \mathrm{NaCl}\end{array}$ \\
\hline $\begin{array}{l}1 \\
2 \\
3 \\
4\end{array}$ & $\begin{array}{r}176 \\
97 \\
122 \\
310\end{array}$ & $\begin{array}{l}1,5 \\
0 \\
0,5 \\
4,0\end{array}$ & $\begin{array}{l}25 \\
13 \\
31 \\
75\end{array}$ \\
\hline
\end{tabular}

Tab. 4. Vergleich der Infektiosität im Überstand von Platten, die gemäß dem Standard-Test-Verfahren beimpft wurden, vor und nach Zugabe von $1 / 10$ Volumen 5-m. $\mathrm{NaCl}$ (2. und 3. Spalte). Die 1. Spalte (1. Test) gibt die im Standard-TestVerfahren ermittelte Plaque-Zahl der entsprechenden Überstände.

Die Infektion von Gewebekulturzellen durch RNS verläuft demnach mit mangelnder Ausbeute. In Versuchen mit ${ }^{32} \mathrm{P}$ markierter RNS gewonnene vorläufige Ergebnisse zeigen, daß ein Teil der RNS reversibel an die Zellen gebunden wird und durch Waschen der Kulturen wieder entfernt werden kann.

Der Unterschied des Infektionsgrades der RNS zwischen 1. und 2. Testung könnte demnach weitgehend durch die im Verlaufe der Manipulationen eintretende Verdünnung der RNS-Lösung und durch reversible Adsorption der RNS an Zellen im 1. Test bedingt sein. Es besteht die Möglichkeit, daß nur ein geringer Teil der potentiell infektiösen RNS im 1. Test durch $1 \cdot 20$ min Kontakt von $4 \cdot 10^{6}$ Zellen mit $0,1 \mathrm{ml}$ RNS-Lösung erfaßt wird. Weitere Versuche zur Klärung des Ausmaßes der Verdünnung der RNS zwischen 1. und 2. Test und der Größe der reversiblen Adsorption von RNS an Zellen sollten zeigen, ob der unterschiedliche Infektiositätsgrad von RNS und intaktem Virus allein durch eine mangelnde Empfänglichkeit der Zellen bedingt ist.

\section{Diskussion}

Die beschriebenen Befunde erlauben eine teilweise Beantwortung der eingangs aufgeworfenen Fragen. Die nach längerem Kontakt mit Zellen von diesen nicht aufgenommene RNS zeigt die gleiche Stabilität wie unbehandelte RNS. Die Frage nach der Inakti- 
vierung der RNS nach Zugabe zu den Zellen durch vorhandene Ribonuclease kann daher verneint werden.

Die Frage nach der Heterogenität der RNS in bezug auf ihr Reaktionsvermögen mit Zellen muß nach wie vor offen bleiben. Es ist uns noch nicht gelungen, mit RNS eine in der Höhe vergleichbare Infektiosität im Plaque-Test zu erreichen, wie sie von intaktem Virus gezeigt wird. Durch einen Vergleich der Infektiosität ${ }^{32} \mathrm{P}$ markierter RNS-Einheit und der Abhängigkeit der Infektiosität der RNS von der Salzkonzentration vor und nach dem ersten Kontakt mit Zellen versuchen wir, weitere Hinweise zu dieser Frage zu gewinnen.

Unsere schon früher aufgeworfene Hypothese von der geringen Empfänglichkeit der Gewebekulturzellen für isolierte RNS unter unseren Testbedingungen findet in den gewonnenen Ergebnissen eine weitere Bestätigung. Da die Hypertonie der RNS-Lösung nach Zugabe zu Zellkulturen von diesen herabgesetzt wird, und andererseits die Infektiosität der RNS stark abhängig ist von der Hypertonie, wird nur ein Teil der potentiell infektiösen RNS nachweisbar. Dieser Befund liefert zugleich auch einen wesentlichen Beitrag zum Verständnis der Kinetik der Induktion von Plaques durch RNS. Wie berichtet ${ }^{6}$, nimmt die Anzahl der durch RNS induzierten Plaques unmittelbar nach Zugabe der RNS zu den Zellen sehr rasch zu. Das weitere Ansteigen der Plaquezahl verlangsamt sich mit der Zeit mehr und mehr. Dieses Verhalten der Kinetik der Plaqueinduktion könnte durch die laufende Verdünnung der hypertonen Salzlösung hervorgerufen werden.

Herrn Prof. Dr. H. Petre und Frau Dr. E. Pette danke ich für ihr Interesse und die Förderung dieser Arbeit. Frau G. Lange sei für gewissenhafte technische Assistenz bedankt. Die Arbeit wurde durchgeführt mit Unterstützung der Deutschen Forschungs. gemeinschaft und des Vereins zur Förderung der Erforschung und Bekämpfung der spinalen Kinderlähmung, Bielefeld.

\title{
Die Wirkung von Stickstoff- und Glucosemangel auf das Teilungsverhalten einer Saccharomyces cerevisiae*
}

\author{
Von E. Greve \\ Aus dem Forstbotanischen Institut der Universität Freiburg im Breisgau \\ (Direktor: Prof. Dr. Dr. H. MARQUaRdT) \\ (Z. Naturforschg. 15 b, 661-666 [1960] ; eingegangen am 29. März 1960)
}

Kulturen des Hefestammes Saccharomyces cerevisiae var. ellips. Yeast Foam (Winge) wurden verschiedenen Ernährungsbedingungen ausgesetzt und das Teilungsverhalten beobachtet.

In synthetischem Minimal-Medium vermehren sich die Zellen auf etwa $15 \%$ der in Bierwürze gemessenen Menge. Werden dem Minimal-Medium die Stickstoffquellen oder die Glucose entzogen, beträgt die Ausbeute nur noch $10 \%$ von der in normalem Minimal-Medium.

Im normalen Minimal-Medium und im N-Mangel-Medium beginnen die Zellen sofort zu knospen und haben die Vermehrung nach 24 Stdn. im wesentlichen abgeschlossen. Bei Glucose-Mangel beginnt die Knospung erst nach ca. 12 Stdn. und ist nach 48 Stdn. abgeschlossen.

Werden Zellen einer 24. Stdn. alten Bierwürzekultur, die sich in exponentiellem Wachstum befinden, für 2 Stdn. dem Minimal- bzw. Mangelmedium ausgesetzt bei gleichzeitiger Kältebehandlung, so beginnen sie sich nach Rücksetzung in Bierwürze sofort und synchron zu teilen, bei Glucose-Mangel etwas schlechter als in den beiden anderen Kulturen.

4 Tage alte, ruhende Bierwürzkulturen, die 2 Stdn. bei Kälte im Stickstoff- oder Glucose-Mangelmedium gehalten wwurden, begannen nach Rücksetzung in Bierwürze nur sehr verzögert zu knospen.

Nach 3 Tagen Minimal- oder Mangelkultur bei normaler Temperatur war der Teilungswiederbeginn in Bierwürze auch verzögert und weniger synchron.

In zahlreichen Arbeiten wurden Hefen unter die verschiedensten Kulturbedingungen gebracht und dann bestrahlt. Dabei kam es zu den widersprechendsten Ergebnissen (Greve 1959 ${ }^{1}$ ). Bei diesen Versuchen interessierte uns vor allem der Einfluß

* Mit Unterstützung der De u t s chen Forschungs gemeins chaf t. verschiedener Ernährungsbedingungen. Insbesondere wollten wir die Wirkung von Stickstoff- und Glucose-Mangel prüfen. Um Interpretationsfehler zu vermeiden, untersuchten wir zunächst eingehend den Einfluß dieser Mangelbedingungen auf das Verhal-

1 E. Greve, Z. Bot. 47, 435 [1959]. 